\title{
Assignment of Milk Fat Fatty Acid Propyl Esters by GC-FID Analysis with the Aid of Ag-ion Solid-phase Extraction
}

\author{
Ryo Sasaki ${ }^{1, *}$, Masatoshi Umezawa ${ }^{1}$, Satoru Tsukahara ${ }^{1}$, Takashi Ishiguro", \\ Shinichi Sato ${ }^{2}$ and Yomi Watanabe ${ }^{3}$ \\ ${ }_{1}^{1}$ Miyoshi Oil \& Fat Co., Ltd. (4-66-1, Horikiri, Katsushika-ku, Tokyo 124-8510, JAPAN) \\ 2 Japan Food Research Laboratories (11-10, Nagayama, 6-chome, Tama, Tokyo 206-0025, JAPAN) \\ ${ }^{3}$ Osaka Municipal Technical Research Institute (1-6-50, Morinomiya, Joto-ku, Osaka 536-8553, JAPAN)
}

\begin{abstract}
The recovery of short-chain fatty acids (FAs) in milk fat (MF) is improved when the analysis of the FA composition of MF by gas chromatography (GC) is conducted with the propyl or butyl ester derivatives, instead of the methyl esters. However, this approach complicates the detection of minor FAs, such as the minor positional isomers of $16: 1$, which represent $<0.2 \%$ of the total content. In addition, the standards of these minor esters are not commercially available. In this study, with the aim to identify minor FAs, the fatty acid propyl esters (FAPEs) of MF were fractionated by Ag-ion solid phase extraction $\left(\mathrm{Ag}^{+}-\right.$ SPE) and analyzed by GC using a DB-23 capillary column. FAPEs were successfully fractionated mainly according to the degree of unsaturation by adjusting the elution conditions of the $\mathbf{A g}^{+}-\mathrm{SPE}$, and the minor FAPEs were easily determined without the aid of standard compounds. For example, by comparison of the GC profile of the saturated $\mathrm{Ag}^{+}$-SPE fraction with that of the original MF, minor FAs, such as iso-, anteiso-, and saturated FAs of 15:0 and 17:0, were expected to be eluted in this order. In addition, 16:1 propyl ester was co-eluted with iso 17:0 propyl ester under the GC conditions used in this study, as confirmed by the detection of the corresponding molecular ions (296 and 312, respectively) by GC-MS. Moreover, 9c,11tconjugated linoleic acid was found to elute between 18:3 and 20:0. To the best of our knowledge, this is the first report suggesting that the peak observed before that of cis-12:1 corresponds to trans-12:1. In conclusion, $\mathrm{Ag}^{+}$-SPE fractionation of FAPEs contributed to the identification of minor FAs in MF without the use of standard compounds.
\end{abstract}

Key words: milk fat, fatty acid composition, Ag-ion solid phase extraction, fatty acid propyl ester, DB-23

\section{INTRODUCTION}

Milk fat (MF) contains many types of fatty acids (FAs), including short-chain (C4-C8) and branched-chain FAs, which are rare in vegetable oils. The FA composition analysis of fats and oils has commonly been conducted through the identification of their methyl ester derivatives ${ }^{1,2)}$ by gas chromatography (GC). However, the methyl esters of short-chain FAs in MF are known to be partly lost during sample preparation, and can therefore be miscalculated ${ }^{33}$. For accurate quantification, derivatization to propyl or butyl esters has been reported to be more suitable than methyl esterification ${ }^{3)}$. In fact, our analysis of the FA composition of MF after methyl and propyl esterification confirmed that the recovery of short-chain fatty acids methyl esters (FAMEs) was low whereas that of their propyl ester derivatives (FAPEs) was sufficient, as previously reported. However, a problem arose during the GC analysis of FAPEs. Because of the peak broadening caused by the increase in the FA esters carbon number, the peak resolution of the FA propyl or butyl esters was poor as compared with that of their methyl derivatives. Thus, minor and branched-chain FAs were difficult to identify without their corresponding standard compounds, which, unlike FAME standards, were not commercially available. In a previous report on the FA composition analysis of MF using butyl esters ${ }^{3)}$, the identification and quantification of branched-chain FAs were not described. Hence, this study aimed to separate and identify the minor FAPEs in MF by GC-FID analysis. Because of the

\footnotetext{
*Correspondence to: Ryo Sasaki, Miyoshi Oil \& Fat Co., Ltd., 4-66-1, Horikiri, Katsushika-ku, Tokyo 124-8510, JAPAN

E-mail: sasakir@so.miyoshi-yushi.co.jp

Accepted September 29, 2015 (received for review May 25, 2015)

Journal of Oleo Science ISSN 1345-8957 print / ISSN 1347-3352 online

http://www.jstage.jst.go.jp/browse/jos/ http://mc.manusriptcentral.com/jjocs
} 
lack of FAPE standards, MF FAPEs were fractionated according to their degree of unsaturation using the Ag-ion solid phase extraction $\left(\mathrm{Ag}^{+}\right.$-SPE $)$method, and the obtained fractions were used for the determination of minor FAPEs. The $\mathrm{Ag}^{+}$-SPE method had originally been developed to fractionate mainly FAMEs according to their degree of unsaturation $^{3-6)}$. Recently, Goto et al. ${ }^{5)}$ applied this method to the fractionation of cis- and trans-geometrical isomers of 18:1, 18:2, and 18:3 methyl esters. We thus used a modified version of this technique to fractionate MF FAPEs and, to the best of our knowledge, this is its first application in this process. The resulting fractions were used for the determination of each MF FAPE by GC-FID, using the medium polar DB-23 (50\% cyanopropyl-methylpolysiloxane) column, in contrast to the previous report ${ }^{3)}$ using a DB-Wax (polyethylene glycol) column. The DB-23 column is one of the most widely used columns for FA composition analysis and proved to be suitable for FA analysis of MF (manuscript in preparation).

\section{EXPERIMENTAL}

\subsection{Materials}

Milk fat was kindly provided by ADEKA Co. (Tokyo, Japan) and Morinaga Milk Industry Co. (Tokyo, Japan). $\mathrm{Ag}^{+}$ -SPE cartridges $(750 \mathrm{mg} / 6 \mathrm{~mL}$ ) were purchased from Sigma-Aldrich (Steinheim, Germany). Hexane, acetone, acetonitrile, acetic acid, and potassium hydroxide were obtained from Kanto Chemical Co.(Tokyo, Japan), and 1-propanol from Wako Pure Chemical Industries (Osaka, Japan). GLC-110 mixture containing anteiso-15:0, anteiso-17:0, and saturated 15:0 were purchased from Matreya LLC (State College, USA). Methyl $9 c, 11 t$-conjugated linoleate was obtained from Nu-Chek Prep, Inc. (Elysian, USA). All other chemicals and solvents were of analytical grade.

\subsection{Preparation of Fatty Acid Propyl Esters (FAPEs)}

One hundred milligrams of MF was dissolved in $2 \mathrm{~mL}$ of hexane. Next, $0.5 \mathrm{~mL}$ of $2.5 \% \mathrm{KOH}$ in 1-propanol was added and the solution was shaken for 1 min using a vortex mixer at room temperature. The mixture was treated with $15 \mu \mathrm{L}$ of acetic acid, shaken for $1 \mathrm{~min}$, and washed with saturated $\mathrm{NaCl}$ solution. After centrifugation at $3000 \mathrm{rpm}$ for $10 \mathrm{~min}$, the hexane phase was recovered and dried over sodium sulfate. This solution was subjected to GC for analysis of the FA composition of total MF or to $\mathrm{Ag}^{+}$-SPE for the fractionation of FAPEs. Ten milligrams of GLC-110 mixture or methyl $9 c, 11 t$-conjugated linoleate were similarly propylated at a half scale.

\section{3 $\mathrm{Ag}^{+}$-SPE fractionation}

Fractionation was conducted following the procedure provided by the manufacturer ${ }^{4)}$ with some modifications.
Before use, the cartridge was conditioned with $4 \mathrm{~mL}$ of acetone followed by $4 \mathrm{~mL}$ of hexane. About $0.1 \mathrm{~mL}$ of FAPEs solution containing approximately $4 \mathrm{mg}$ of FAPEs, prepared as described in the previous section, was loaded onto the column. Elution was performed with $6 \mathrm{~mL}$ of a mixture of hexane and acetone (96:4, v/v; Fraction 1 (Fr1)), $2 \mathrm{~mL}$ of another hexane/acetone mixture (90:10, v/ v; Fr2), $2 \mathrm{~mL}$ of a third hexane/acetone mixture (90:10, v/v; Fr3), $2 \mathrm{~mL}$ of acetone(Fr4), $2 \mathrm{~mL}$ of acetone(Fr5), and finally with $5 \mathrm{~mL}$ of a mixture of acetone and acetonitrile $(3: 2, \mathrm{v} / \mathrm{v} ; \mathrm{Fr} 6)$. All fractions were directly transferred to the GC vial and sent for GC analysis without concentration, to avoid the loss of volatile propyl esters of short-chain FAs.

\subsection{Gas Chromatography with Flame Ionization Detector (GC-FID)}

GC analysis was carried out on a GC-2025 gas chromatograph (Shimadzu Co., Kyoto, Japan) equipped with a flame ionization detector(FID). The temperatures of the injector and detector were set at $250^{\circ} \mathrm{C}$. Helium was used as a carrier gas at the flow rate of $1 \mathrm{~mL} / \mathrm{min}$. The injection volume was $1 \mu \mathrm{L}$ for total MF and $2 \mu \mathrm{L}$ for the fractionated samples obtained by $\mathrm{Ag}^{+}$-SPE. The split ratio was set at 50:1 for total MF and at 20:1 for fractionated samples. A capillary column DB-23 (Agilent Technologies, Santa Clara, CA, USA; $30 \mathrm{~m} \times 0.25 \mathrm{~mm}, 0.25 \mu \mathrm{m}, 50 \%$ cyanopropylmethylpolysiloxane) was used. The oven temperature was programmed as follows: $80^{\circ} \mathrm{C}$ for $3 \mathrm{~min}$, increased to $180^{\circ} \mathrm{C}$ at the rate of $8^{\circ} \mathrm{C} / \mathrm{min}$, further raised to $240^{\circ} \mathrm{C}$ at the rate of $3^{\circ} \mathrm{C} / \mathrm{min}$, and held at $240^{\circ} \mathrm{C}$ for $5 \mathrm{~min}$. All analyses were conducted three times and mean values were presented.

\subsection{Gas Chromatography-Mass Spectrometry (GC-MS)}

GC-MS was carried out on a Trace 1310 coupled with a TSQ 8000 (Thermo Fisher Scientific Inc., Waltham, MA, USA). The temperatures of the injector and transfer lines were set at $250^{\circ} \mathrm{C}$, and the ion source temperature was set at $200^{\circ} \mathrm{C}$. Analysis in the full scan mode covered the range of m/z 50-450. A capillary column TC-70 (GL Sciences Inc., Tokyo Japan; $60 \mathrm{~m} \times 0.25 \mathrm{~mm}, 0.25 \mu \mathrm{m}, 70 \%$ cyanopropyl equivalent-silphenylene-siloxane) was used. Other GC conditions were the same as those used for GC-FID.

\section{RESULTS AND DISCUSSION}

\subsection{Separation of MF FAPEs by Ag-ion SPE}

In this study, we attempted to assign the peaks in the gas chromatogram obtained using a DB-23 capillary column to the minor FAPEs in MF, without the aid of the corresponding commercial standards. For this purpose, the FAPEs of $\mathrm{MF}$ were first fractionated by $\mathrm{Ag}^{+}-\mathrm{SPE}$. The original protocol for the separation of FAMEs according to the degree of 
unsaturation by $\mathrm{Ag}^{+}$-SPE was as follows ${ }^{6)}$. FAMEs (1 mg) in hexane were loaded onto the SPE column, which had been previously preconditioned with $4 \mathrm{~mL}$ of acetone and $4 \mathrm{~mL}$ of hexane, sequentially. FAMEs were fractionated into four fractions by sequential elution with $6 \mathrm{~mL}$ of hexane/ acetone 96:4 (Fr1; mainly containing saturated FAs and trans-monounsaturated FAs), $4 \mathrm{~mL}$ of hexane/acetone 90:10 (Fr2; containing cis-monounsaturated, and cis/transor trans/cis-conjugated linoleic acids (CLAs)), $4 \mathrm{~mL}$ of acetone (Fr3; containing cis-diunsaturated FAs), and $5 \mathrm{~mL}$ of acetone/acetonitrile 3:2 (Fr4; containing triunsaturated FAs). The resulting fractions were evaporated at $40^{\circ} \mathrm{C}$ under a $\mathrm{N}_{2}$ gas stream and brought to $1 \mathrm{~mL}$ by addition of hexane prior to GC analysis. Two major disadvantages were found in the direct application of the original protocol ${ }^{6}$ to the determination of FAPEs in MF. The first problem was that short-chain FAs were not detected, presumably because of their volatilization during the concentration step. However, without concentration, the minor peaks (about $1 \mathrm{ppm}$ in GC sample solution of MF FAPEs)were hardly detected under the applied GC-FID or GC-MS conditions. The second problem was that, increasing the amount of sample loaded onto the SPE column, the signals of the minor peaks increased but the resolution of the fractionation decreased because of the carry-overs from previous fractions. Thus, the protocol was modified as follows. Approximately $4 \mathrm{mg}$ of FAPEs in hexane was loaded onto the SPE column, preconditioned as described above. The FAPEs were fractionated into six fractions by sequential elution with $6 \mathrm{~mL}$ of hexane/acetone 96:4 (Fig. 1, Fr1; containing mainly saturated FAs and trans-monounsaturated
FAs), 2 mL of hexane/acetone 90:10 (Fig. 5, Fr2; containing carry-overs from Fr1 and cis-monounsaturated FAs), $2 \mathrm{~mL}$ of hexane/acetone 90:10 (Fig. 1, Fr3; containing cis-monounsaturated, and cis/trans-CLAs), $2 \mathrm{~mL}$ of acetone (Fig. 5, Fr4; containing carry-overs from Fr3 and cis-diunsaturated FAs), 2 mL of acetone (Fig. 1, Fr5; containing cis-diunsaturated FAs), and $5 \mathrm{~mL}$ of acetone/acetonitrile 3:2 (Fig. 1, Fr6; containing triunsaturated FAs). Each fraction was directly sent for GC analysis without any concentration step. Following this protocol, FAPEs were successfully fractionated according to the degree of unsaturation, avoiding carry-overs. Similar fractionation profiles were obtained repeating the analyses.

\subsection{Assignment of FAPEs in the region between 14:0 and 18:0}

The chromatograms of original MF and of the sample fractionated by $\mathrm{Ag}^{+}$-SPE are shown in Fig. 1. The major peaks of FAs, such as saturated, mono-, and di-unsaturated FAs, in Fr1, Fr3, and Fr5 were easily assigned. In order to assign the peaks of minor FAs and the overlapping peaks, essential fractions of FAPEs were also analyzed by GC-MS connected to a TC-70 column to confirm their molecular weights (MWs). Besides CLA in Fr3, for each fraction obtained by $\mathrm{Ag}^{+}$-SPE, the elution order of FAs in the chromatograms obtained with a DB-23 and with a TC-70 column was consistent; i.e., the two chromatograms were comparable. The chromatogram of FAPEs from the DB-23 column shows branched or positional isomers of unsaturated FAs in the region between 14:0 and 18:0(Fig. 2). The total ion current (TIC) chromatogram and MS spectrum of

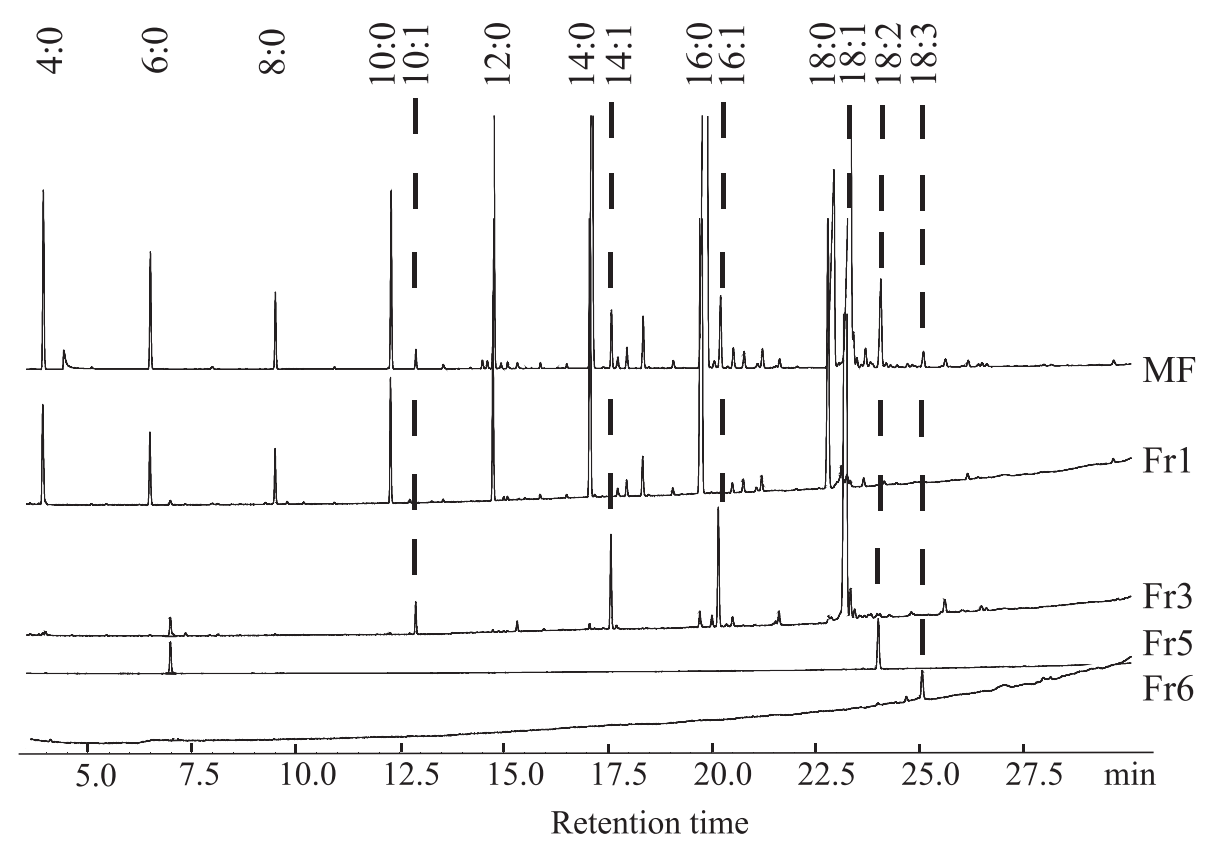

Fig. 1 Gas chromatograms of MF FAPEs and their fractionated samples by $\mathrm{Ag}^{+}$-SPE using a DB-23 column. MF, total milk fat; Fr1, saturated FAs; Fr3, mono- unsaturated FAs; Fr5, di-unsaturated FAs; Fr6, tri-unsaturated FAs. 


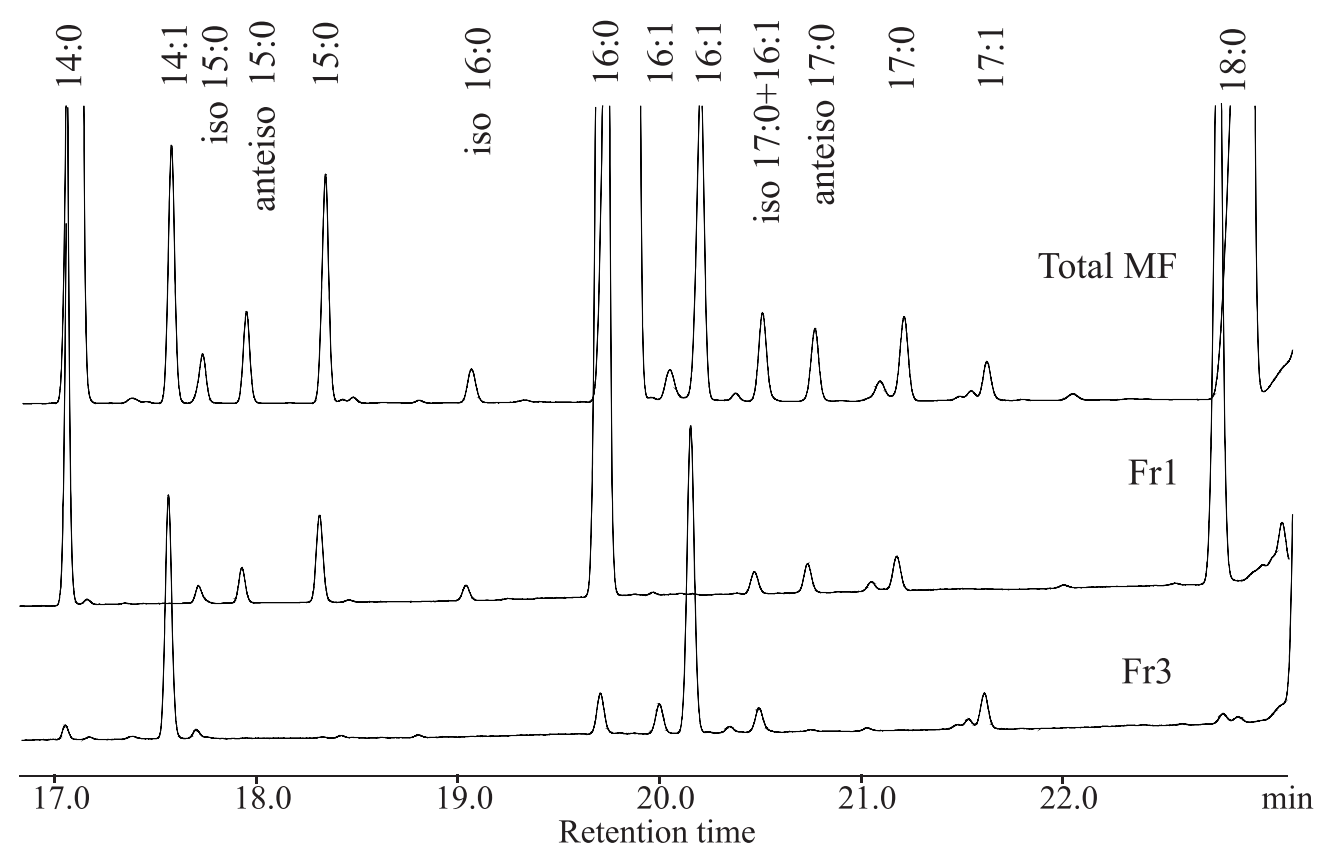

Fig. 2 Partial chromatogram of MF in the region of 14:0 to 18:0 using a DB-23 column. From the top: total milk fat, Fr1 (saturated fraction), Fr3 (mono-saturated fraction).
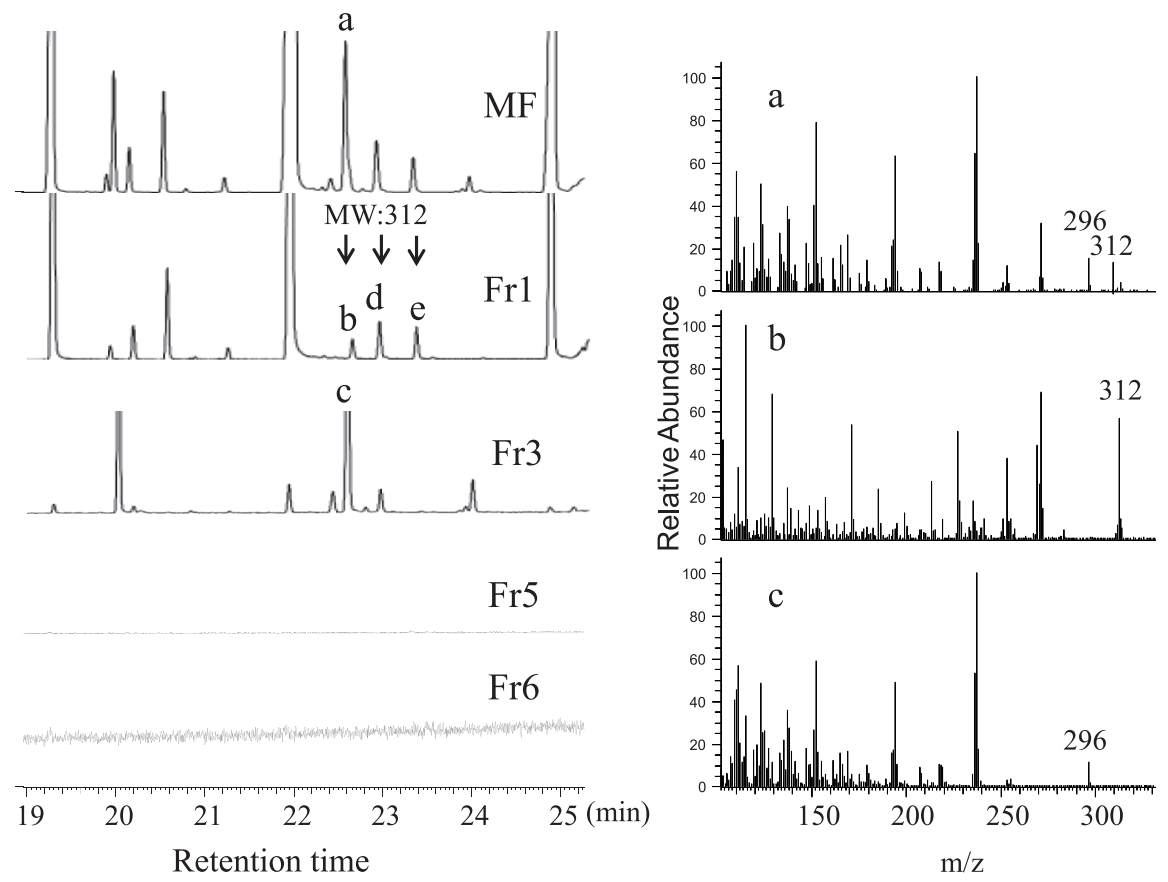

Fig. 3 Partial TIC chromatogram of MF FAPEs in the region of 14:0 to 18:0 using a TC-70 column (left). Fraction numbers are the same as in Fig. 1. MS spectrum of peak a, b and c (right).

the same region are illustrated in Fig. 3. The fatty acid chain lengths were confirmed by the MWs revealed by GC-MS. The three peaks b, d, and e in Fr1 (Fig. 3) showed identical molecular weight (312), indicating that they are isomers of 17:0 FA. The elution order of 17:0 isomers was reported to be iso $17: 0$, anteiso $17: 0$, and saturated $17: 0^{7,8)}$; thus, peaks b, d, and e are attributed to those isomers, respectively. Other branched-chain FAs, such as iso 15:0, anteiso 15:0, and saturated 15:0, were assigned on the basis of the MWs and of the retention times, and were comparable to the corresponding propyl esters, obtained by propylation of the standard GLC-110 mixture that in- 
Table 1 The assignment of FAPEs separated by DB-23 and TC-70 in the region of 14:0 to 18:0.

\begin{tabular}{|c|c|c|c|c|c|c|}
\hline \multicolumn{3}{|c|}{ DB-23 } & \multicolumn{4}{|c|}{ TC-70 } \\
\hline FAPE & $\mathrm{RT}^{\mathrm{a}}$ & $\mathrm{Fr}^{\mathrm{b}}$ & FAPE & $\mathrm{m} / \mathrm{z}^{\mathrm{c}}$ & RT & $\mathrm{Fr}$ \\
\hline $14: 0$ & 17.13 & 1 & $14: 0$ & 270 & 19.30 & 1 \\
\hline 14:1 & 17.59 & 3 & iso $15: 0$ & 284 & 19.90 & 1 \\
\hline iso $15: 0$ & 17.75 & 1 & $14: 1$ & 268 & 19.99 & 3 \\
\hline anteiso $15: 0$ & 17.96 & 1 & anteiso $15: 0$ & 284 & 20.16 & 1 \\
\hline $15: 0$ & 18.36 & 1 & $15: 0$ & 284 & 20.55 & 1 \\
\hline iso $16: 0$ & 19.08 & 1 & iso $16: 0$ & 298 & 21.23 & 1 \\
\hline $16: 0$ & 19.89 & 1 & $16: 0$ & 298 & 22.03 & 1 \\
\hline 16:1 & 20.06 & 3 & 16:1 & 296 & 22.42 & 3 \\
\hline $16: 1 \mathrm{n}-7$ & 20.22 & 3 & $16: 1 \mathrm{n}-7$ & 296 & 22.59 & 3 \\
\hline 16:1 & 20.52 & 3 & iso $17: 0$ & 312 & 22.59 & 1 \\
\hline iso $17: 0$ & 20.52 & 1 & $16: 1$ & 296 & 22.94 & 3 \\
\hline anteiso $17: 0$ & 20.78 & 1 & anteiso $17: 0$ & 312 & 22.94 & 1 \\
\hline $17: 0$ & 21.23 & 1 & $17: 0$ & 312 & 23.45 & 1 \\
\hline $17: 1$ & 21.64 & 3 & $17: 1$ & 310 & 23.98 & 3 \\
\hline 18:0 & 22.94 & 1 & 18:0 & 326 & 24.93 & 1 \\
\hline
\end{tabular}

${ }^{\mathrm{a}} \mathrm{RT}$ : retention time.

${ }^{\mathrm{b}} \mathrm{Fr}$ : The number of fraction from the $\mathrm{Ag}^{+}-\mathrm{SPE}$.

${ }^{\mathrm{c}} \mathrm{m} / \mathrm{z}$ : molecular ion $[\mathrm{M}]^{+}$of the FAPE obtained by GC-MS.

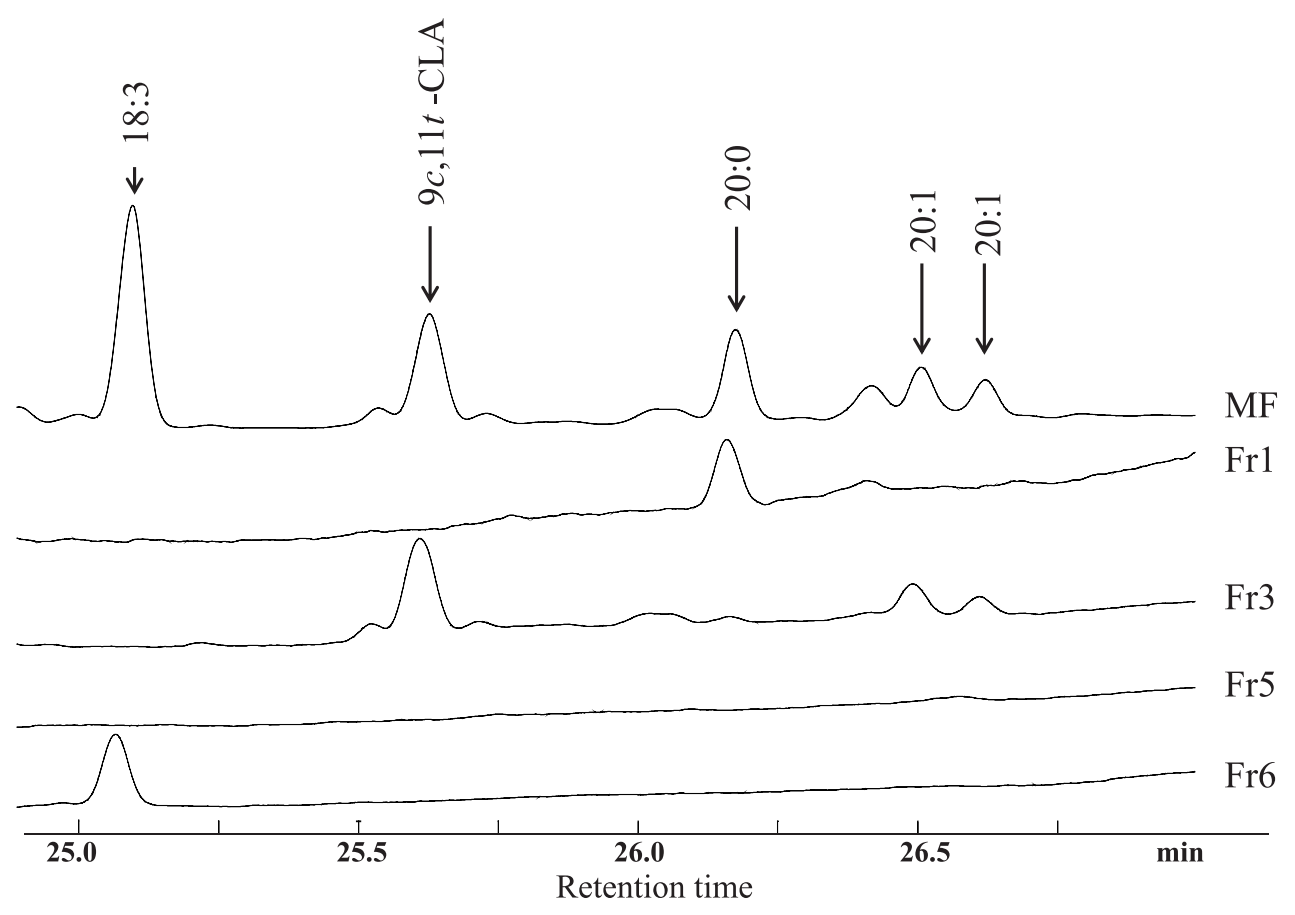

Fig. 4 Partial chromatogram of MF in the region of 18:3 to 20:1 using a DB-23 column. Fraction numbers are the same as in Fig. 1. 
Table 2 Fatty acid profile of MF analyzed as FAPE by DB-23 column.

\begin{tabular}{|c|c|c|c|}
\hline FAPE & Retention time & $\begin{array}{c}\text { Sample 1 } \\
(\text { area } \% \pm \text { standard deviation })\end{array}$ & $\begin{array}{c}\text { Sample } 2 \\
(\text { area } \% \pm \text { standard deviation })\end{array}$ \\
\hline $4: 0$ & 3.93 & $2.75 \pm 0.055$ & $3.41 \pm 0.010$ \\
\hline $6: 0$ & 6.50 & $1.85 \pm 0.033$ & $2.13 \pm 0.003$ \\
\hline $8: 0$ & 9.51 & $1.17 \pm 0.014$ & $1.27 \pm 0.002$ \\
\hline $9: 0$ & 10.93 & $0.03 \pm 0.003$ & $0.03 \pm 0.001$ \\
\hline $10: 0$ & 12.29 & $2.74 \pm 0.028$ & $2.97 \pm 0.007$ \\
\hline $10: 1$ & 12.88 & $0.29 \pm 0.002$ & $0.28 \pm 0.000$ \\
\hline $11: 0$ & 13.55 & $0.07 \pm 0.010$ & $0.09 \pm 0.022$ \\
\hline $12: 0$ & 14.77 & $4.28 \pm 0.025$ & $3.40 \pm 0.005$ \\
\hline trans-12:1 & 15.09 & $0.10 \pm 0.007$ & $0.10 \pm 0.018$ \\
\hline $12: 1$ & 15.33 & $0.12 \pm 0.003$ & $0.11 \pm 0.018$ \\
\hline $13: 0$ & 15.88 & $0.09 \pm 0.002$ & $0.09 \pm 0.003$ \\
\hline iso $14: 0$ & 16.52 & $0.06 \pm 0.001$ & $0.11 \pm 0.004$ \\
\hline $14: 0$ & 17.13 & $10.94 \pm 0.006$ & $11.27 \pm 0.025$ \\
\hline $14: \ln -9$ & 17.59 & $0.99 \pm 0.005$ & $0.90 \pm 0.003$ \\
\hline iso $15: 0$ & 17.75 & $0.22 \pm 0.002$ & $0.25 \pm 0.007$ \\
\hline anteiso 15:0 & 17.96 & $0.36 \pm 0.003$ & $0.47 \pm 0.001$ \\
\hline $15: 0$ & 18.36 & $0.92 \pm 0.005$ & $1.01 \pm 0.003$ \\
\hline iso $16: 0$ & 19.08 & $0.16 \pm 0.002$ & $0.21 \pm 0.007$ \\
\hline 16:0 & 19.89 & $33.10 \pm 0.181$ & $30.00 \pm 0.058$ \\
\hline $16: 1$ & 20.06 & $0.18 \pm 0.013$ & $0.18 \pm 0.021$ \\
\hline $16: \ln -7$ & 20.22 & $1.48 \pm 0.016$ & $1.29 \pm 0.021$ \\
\hline iso $17: 0+16: 1$ & 20.52 & $0.42 \pm 0.005$ & $0.45 \pm 0.002$ \\
\hline anteiso 17:0 & 20.78 & $0.35 \pm 0.004$ & $0.42 \pm 0.004$ \\
\hline $17: 0$ & 21.23 & $0.43 \pm 0.004$ & $0.49 \pm 0.001$ \\
\hline $17: 1$ & 21.64 & $0.19 \pm 0.005$ & $0.19 \pm 0.010$ \\
\hline $18: 0$ & 22.94 & $8.65 \pm 0.036$ & $10.67 \pm 0.023$ \\
\hline $18: 1^{* 1}$ & 23.35 & $21.78 \pm 0.020$ & $22.11 \pm 0.044$ \\
\hline $18: 1^{* 2}$ & 23.41 & $0.67 \pm 0.063$ & $0.56 \pm 0.020$ \\
\hline $18: 2$ n-6 & 24.07 & $2.34 \pm 0.027$ & $2.40 \pm 0.007$ \\
\hline $18: 3$ n-3 & 25.10 & $0.39 \pm 0.006$ & $0.33 \pm 0.004$ \\
\hline $9 c, 11 t$-CLA & 25.63 & $0.22 \pm 0.005$ & $0.49 \pm 0.011$ \\
\hline $20: 0$ & 26.17 & $0.18 \pm 0.032$ & $0.13 \pm 0.007$ \\
\hline $20: 1$ & 26.51 & $0.09 \pm 0.005$ & $0.10 \pm 0.003$ \\
\hline $20: 1$ & 26.62 & $0.07 \pm 0.008$ & $0.03 \pm 0.002$ \\
\hline $22: 0$ & 29.67 & $0.13 \pm 0.005$ & $0.05 \pm 0.005$ \\
\hline Unidentified $^{* 3}$ & & 2.19 & 2.01 \\
\hline total & & 100 & 100 \\
\hline
\end{tabular}

${ }^{* 1}$ consists mainly of cis-18:1n-9

${ }^{* 2}$ consists mainly of cis-18:1n-7

${ }^{* 3}$ unidentified FAPEs include 18:1 and 18:2 configurational or positional isomers, and minor peaks that were not confirmed by GC-MS. 


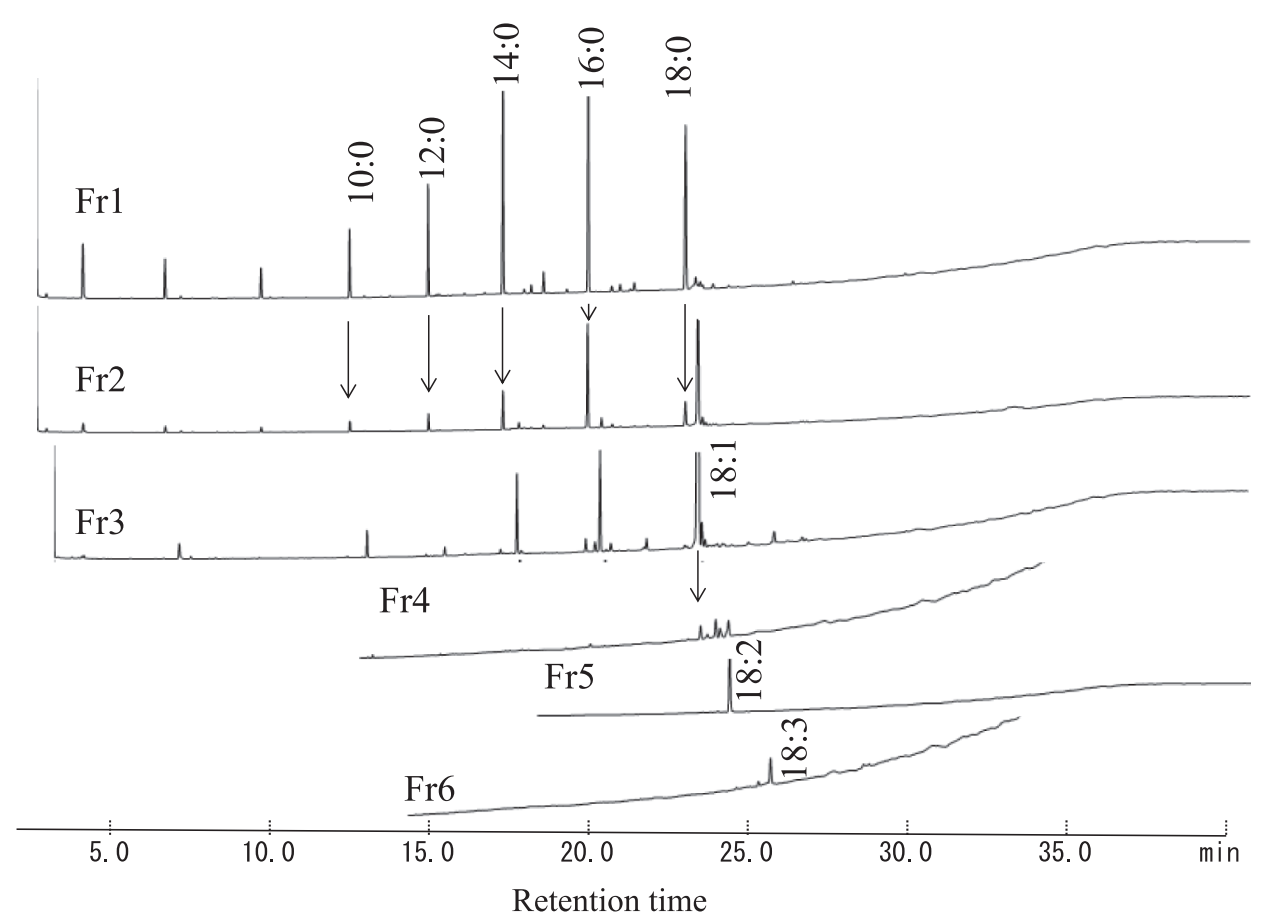

Fig.5 GC chromatogram of fractionated FAPE by $\mathrm{Ag}^{+}$-SPE. From the top to the bottom, Fr1 to Fr6. Carry-overs were indicated with arrows.

cluded anteiso 15:0, anteiso 17:0, and saturated 15:0. Without $\mathrm{Ag}^{+}$-SPE fractionation of MF FAPEs, the abovementioned assignment of the peaks would have been difficult because of the complicated chromatograms.

The assignment of the peaks overlapping because of coelution was achieved by the combination of $\mathrm{Ag}^{+}-\mathrm{SPE}$ fractionation and GC-MS analysis. Peak a, which is a single peak in the chromatogram of the original $\operatorname{MF}$ (Fig. 3, a), was divided into two peaks (Fig. 3, b, c) after $\mathrm{Ag}^{+}$-SPE fractionation. GC-MS analysis showed that these two peaks have different MWs (312 and 296, respectively) (Fig. 3) and could therefore be assigned to iso 17:0 and 16:1 n-7, using the TC-70 column. Likewise, other minor (iso 16:0) and overlapping peaks (minor isomer of 16:1 and iso 17:0 on the DB23 column; minor isomer of $16: 1$ and anteiso 17:0 on the TC-70 column) were assigned. The assignment of the MF FAPEs analyzed using a DB-23 and a TC-70 column in the region between 14:0 and 18:0 is summarized in Table 1.

\subsection{Assignment of 18:3, 20:0, 20:1, and CLA}

In the analysis of the FA composition of MF using FAMEs, 18:3, 20:0, 20:1, and CLA have similar retention times ${ }^{1)}$. In this study, fatty acids were derivatized to their propyl esters. The increase in the carbon number as compared with FAMEs was expected to lower the resolution of the GC peaks. Moreover, the elution order of these peaks was dependent on the type(i.e., polarity) of column used. The region of interest (25.00-27.00 min) of the chromatogram recorded using the DB-23 column is shown in Fig. 4.
By comparing the fractions obtained by $\mathrm{Ag}^{+}$-SPE, the peak at 26.17 min was identified as 20:0, because Fr1 consisted of saturated FAs. Fr3, which consisted mainly of monounsaturated FAs, was found to contain CLA. Among its isomers, $9 c, 11 t$-CLA is the most abundant in MF, and was thus estimated to correspond to the peak at $25.63 \mathrm{~min}$ in Fr3. This was also confirmed by its MW, revealed by GC-MS, and by comparing its retention time to the standard propyl $9 c, 11 t$-CLA. This peak was therefore distinguished from the two $20: 1$ peaks at $26.51 \mathrm{~min}$ and 26.62 min in Fr3. The peak at 25.10 min was assigned as 18:3, because it was detected in Fr6, which mainly consisted of triunsaturated FAs. Thus, the order of elution using a DB-23 column was concluded to be as follows: $18: 3,9 c, 11 t$ CLA, 20:0, and 20:1. This order corresponds to that found in a previous study ${ }^{1)}$ using Supelcowax-10 column, which has a medium polar stationary phase (poly (ethylene glycol)). Hence, the DB-23 and Supelcowax-10 columns are likely to show similar chromatographic performance in FA analysis.

\subsection{Assignment of minor peaks, configurations, and posi- tional isomers}

The assignment of FAPEs of MF by $\mathrm{Ag}^{+}$-SPE and GC-MS analysis in two milk fat samples (samples 1 and 2) is summarized in Table 2. In this table, because of the difficulty of preparing propyl ester standards, the direct peak areas (area \%) of each propyl ester can be regarded as semi quantitative values, without response factor correction, 
and therefore do not reflect the real abundance of each derivative. The minor FAPEs (9:0, 11:0, 13:0, and iso 14:0), whose molecular ions were confirmed by GC-MS, are included in Table 2. The peak of trans-12:1 was clearly identified, as it was found in Fr1 and showed the same MS spectrum as cis-12:1. However, the position of the double bond could not be determined. Moreover, the DB-23 column used in this study cannot resolve the configurations and positional isomers of 18:1, 18:2, and 18:3; these isomers were therefore included in the unidentified FAs category in Table 2. For the analysis of trans-FA, high polar columns such as CP-Sil $88^{7)}$ or SLB-IL $111^{9)}$ are recommended.

\section{CONCLUSION}

The analysis of MF FA composition as FAPEs is advantageous for the recovery and identification of short-chain FAs. However, because of the increase in the carbon number, a lower resolution of other minor FAs, such as odd-numbered and branched-chain FAs, was observed as compared with the analysis of FAMEs. In this study, FAPEs of MF were fractionated by $\mathrm{Ag}^{+}$-SPE prior to GC analysis. Most of the minor FAPEs in MF were successfully assigned in the GC-FID chromatogram obtained using a DB-23 column, without the aid of FAPE standards. Moreover, our results suggested that MF contained trans-12:1 in addition to $c i s-12: 1$.

\section{ACKNOWLEDGEMENTS}

The authors thank A. Yamashita (ADEKA Co.), T. Arishima and S. Watanabe (Fuji Oil Co.), J. Imagi, T. Sano, and R. Hori (J-Oil Mills, Inc.), K. Saito and R. Homma (Kao Co.), C. Sato and A. Sasaki (The Nisshin OilliO Group), Y. Miyazaki (NOF Co.), T. Shibuya and Y. Tsukahara (Showa Sangyo Co.), and T. Nagai and K. Yoshinaga (Tsukishima Foods Industry Co.) for their support.

\section{References}

1) Kramer, J. K. G.; Hernandez, M.; Cruz-Hernandez, C. Kraft, J.; Dugan, M. E. R. Combining results of two GC separations partly achieves determination of all cis and trans 16:1, 18:1, 18:2 and 18:3 except CLA isomers of milk fat as demonstrated using Ag-ion SPE fractionation. Lipids 43, 259-273(2008).

2) Vingering, N.; Ledoux, M. Use of BPX-70 60-m GC columns for screening the fatty acid composition of industrial cookies. Eur. J. Lipid Sci. Technol. 111, 669677 (2009).

3) Ulberth, F.; Gabernig, R. G.; Schammel, F. Flame-ionization detector response to methyl, ethyl, propyl, and butyl esters of fatty acids. J. Am. Oil. Chem. Soc. 76, 263-266 (1999).

4) Christie, W. W. Silver ion chromatography using solidphase extraction columns packed with bonded-sulfonic acid phase. J. Lipid Res. 30, 1471-1473 (1989).

5) Goto, H.; Shionoya, N.; Sugie, M.; Tominaga, M., Shimels, O.; Taniguchi, M.; Igarashi, T.; Hirata, Y. Novel pre-fractionation method of trans fatty acids by gas chromatography with silver-ion cartridge column. $J$. Oleo Sci. 61, 49-56 (2012).

6) SUPELCO Technical Report, Fractionation of cis/ trans FAMEs using Discovery Ag-ion SPE, sigma-aldrich.com/analytical, e-mail: supelco@sial.com, Bellefonte, PA.

7) Kramer, J. K. G.; Blackadar, C. B.; Zhou, J. Evaluation of two GC columns, (60-m SUPELCOWAX 10 and 100m CP Sil 88) for analysis of milk fat with emphasis on CLA, 18:1, 18:2 and 18:3 isomers and short- and longchain FA. Lipids 37, 823-835 (2002).

8) Schröder, M.; Vetter, M. Detection of 430 fatty acid methyl esters from a transesterified butter sample. $J$. Am. Oil. Chem. Soc. 90, 771-790(2013).

9) Yoshinaga, K.; Asamuma, M.; Mizobe, H.; Kojima, K.; Nagai, T.; Beppu, F.; Gotoh, N. Characterization of cisand trans-octadecenoic acid positional isomers in edible fat and oil using gas chromatography-flame ionization detector equipped with highly polar ionic liquid capillary column. Food Chem. 160, 39-45 (2014). 